\title{
Airway Remodeling in Asthma: Tumor of the Airway?
}

\author{
Chun Y. Seow ${ }^{a, c}$ Stephan van Eeden ${ }^{b, c}$ \\ ${ }^{a}$ Department of Pathology and Laboratory Medicine, ${ }^{b}$ Department of Medicine and \\ 'James Hogg Research Centre, University of British Columbia, Vancouver, B.C., Canada
}

A thickened airway wall with increased airway smooth muscle mass and extracellular matrix deposition as a result of airway remodeling is a hallmark of persistent asthma. The mechanism underlying the remodeling process is not entirely clear. It is thought that the chronic airway inflammation seen in asthma is a major contributor, but anti-inflammatory strategies often do not bring resolution to airway remodeling [1]. Findings reported by Chen et al. [2] in this issue of Respiration explore the effects of the leukotriene inhibitor (zileuton) on airway remodeling in an ovalbumin-sensitized rat model and revealed that downstream pathways of leukotriene stimulation contribute to airway remodeling. Furthermore, the leukotriene signaling appears to follow the same pathways as those considered to be linked to tumorigenesis $[3,4]$. These findings suggest a common mechanism underlying the increase in airway tissue mass seen in chronic asthma and the tumor growth seen in malignancy.

The primary components of asthma treatment separately target acute airway obstruction and chronic airway inflammation. Bronchodilators (predominately $\beta_{2}$ agonists) are used to treat the acute symptoms and corticosteroids are used to control inflammation. Increasingly, antileukotriene agents are being added to inhaled corticosteroids in combination therapy, especially for severe asthma. Antileukotrienes are also used on their own as a frontline therapy, mostly for mild asthma [5]. Antileukotrienes have both anti-inflammatory and bronchodilatory properties and the therapy has been shown to have an acute and sustained beneficial effect on lung function (changes in $\mathrm{FEV}_{1}$ ) in chronic persistent asthma [6]. Antileukotrienes do not have major side effects and appear to be well tolerated by most patients [7], but a limitation of their widespread use may be due to the large percentage of nonresponders [8]. However, for those intolerant or resistant to corticosteroids but responsive to antileukotrienes, treatment with leukotriene inhibitors has become an invaluable therapeutic option.

There are two types of leukotriene inhibitors: the leukotriene receptor antagonists such as montelukast and those that inhibit leukotriene formation such as zileuton. Zileuton is an inhibitor of the enzyme 5-lipoxygenase, which forms leukotrienes from arachidonic acid. The network of signaling pathways associated with leukotrienes is complex and is not completely understood [6]. The findings by Chen et al. [2] have shed light on this subject, specifically with regard to perturbations in the signaling pathways seen in allergen-sensitized animals. They have identified some potentially important downstream molecular players in leukotriene signaling in ovalbumin-sensitized rats with chronic inflammation, thickened airway walls and reduced lung function. They also showed that zileuton significantly reduced the expression of biomarkers of chronic inflammation, significantly reversed the airway remodeling and improved lung function in these sensitized, bronchohyperresponsive rats.

\section{KARGER}

E-Mail karger@karger.com

www.karger.com/res
C 2013 S. Karger AG, Basel

0025-7931/13/0865-0364\$38.00/0
Dr. Stephan van Eeden

James Hogg Research Centre

University of British Columbia

Vancouver, BC V6Z 1 Y6 (Canada)

E-Mail stephan.vaneeden@ @li.ubc.ca 
An earlier study by Burgess et al. [9] pointed out that phosphoinositide 3-kinase (PI3K) could be a key molecule in the hyperplasia response of human airway smooth muscle in asthma. Following this lead, Chen et al. [2] have demonstrated that activation of the PI3K/Akt/mTOR pathway is associated with leukotriene-induced (zileuton-sensitive) airway remodeling in ovalbumin-sensitized rats. The mammalian target for rapamycin (mTOR) is regarded as a master switch of cellular metabolism that regulates cell growth and proliferation. The enzyme complex is made up of two components, mTORC1 and mTORC2 [3]. mTORC1 stimulates protein synthesis by phosphorylating the ribosomal S6 kinase 1 (S6K1) and the eukaryotic translation initiation factor 4E-binding protein 1 (4E-BP1). Phosphorylation of 4E-BP1 leads to the release of eukaryotic initiation factor $4 \mathrm{E}$ (eIF4E) from $4 \mathrm{E}-\mathrm{BP} 1$; formation of eIF4F results from binding of eIF4E with eIF4G (fig. 1). eIF4E itself is known to have antiapoptotic activity [10-12]. eIF4F and signaling from the S6K1 pathway are important in the initiation of mRNA translation that produces proteins for cell growth and cell cycling. mTORC2 activates the serum- and glucocorticoid-regulated kinase and protein kinase $\mathrm{C}$, two important enzymes known to be involved in the regulation of cell cycle and survival. Chen et al. [2] have shown that virtually all enzymes and proteins associated with the $\mathrm{PI} 3 \mathrm{~K} / \mathrm{Akt} / \mathrm{mTOR}$ pathway (fig. 1) are activated in the airways of ovalbumin-sensitized rats, presumably due to leukotriene stimulation (because the level of activation could be attenuated by zileuton). As a result of the hyperactivation, an excess amount of cyclin D1 is produced, which could explain the increased airway smooth muscle mass which they found in the sensitized rats. The broad effects of zileuton in reversing airway wall-thickening and angiogenesis as well as reducing the level of inflammatory mediators and improving lung function are remarkable. Even more remarkable is the finding that the pathways leading to airway remodeling closely overlap those responsible for tumorigenesis $[3,4]$. This raises a question: is airway remodeling a form of hyperplasia or tumor growth? The findings that deficiencyin $\mathrm{C} / \mathrm{EBP}$ (CCAAT/ enhancer binding protein $\alpha$ ) in human asthmatic airway smooth muscle cells [13] could be linked to airway remodeling in vivo and that mutation in the gene encoding $\mathrm{C} / \mathrm{EBPa}$ is linked to some malignancies [14] raise the same question. The outcome of airway remodeling and tumor growth is at least superficially the same: there is an increase of tissue mass. If both processes are the results of disturbance in the signal transduction in the PI3K/Akt/ $\mathrm{mTOR} / \mathrm{C} / \mathrm{EBP} \alpha$ axis of pathways $[15,16]$, then the same

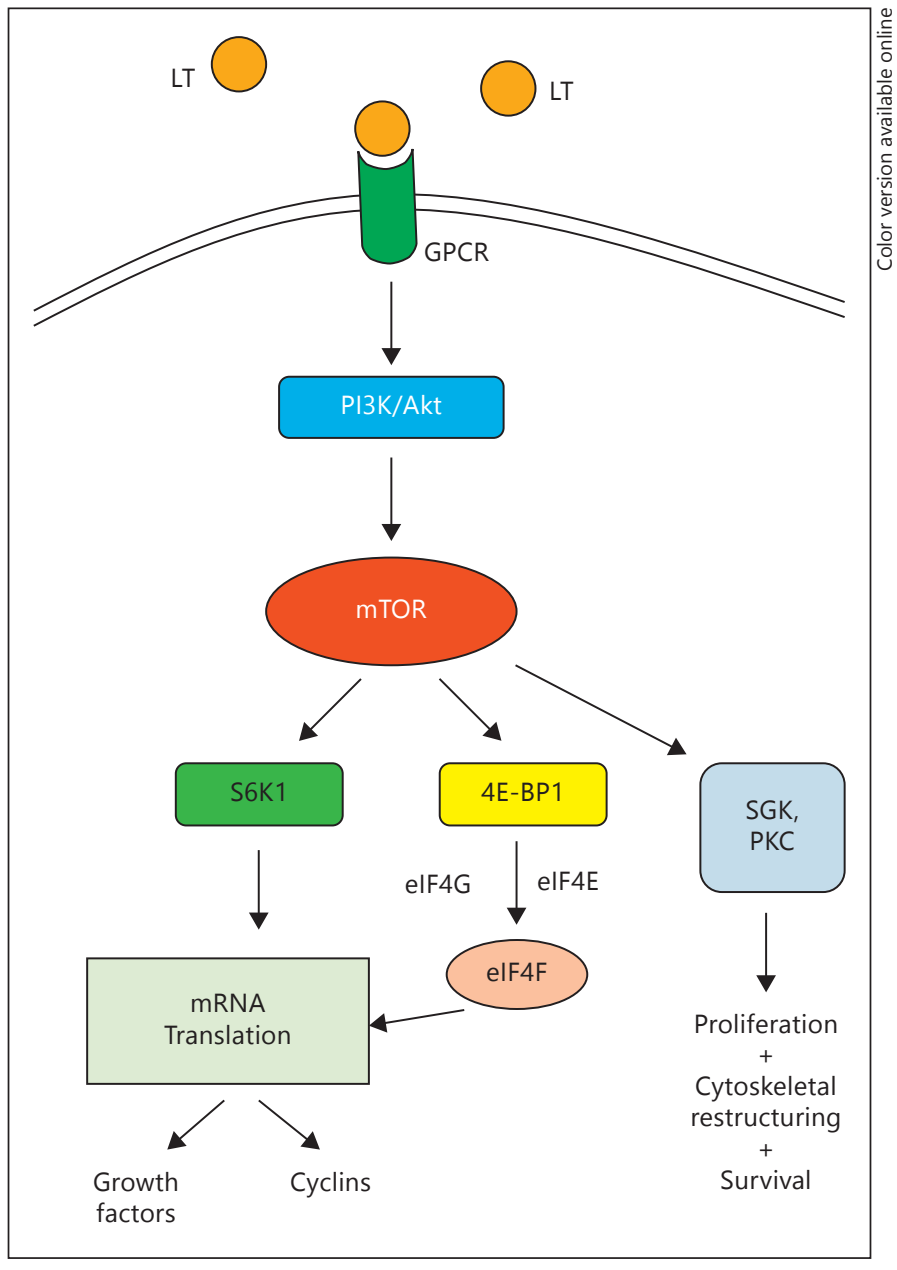

Fig. 1. Canonical pathway for PI3K/Akt/mTOR signaling in cell growth and proliferation. Leukotrienes (LT) act by binding to specific G-protein-coupled receptors (GPCR), such as GPR17, leading to activation of PI3K and phosphorylation of Akt which in turn activates mTOR. mTORC1 activates $\mathrm{S} 6 \mathrm{~K} 1$ and $4 \mathrm{E}-\mathrm{BP} 1$ by phosphorylation. Phosphorylation of 4E-BP1 (an eIF4E binding protein) releases eIF4E which then binds with eIF4G to form eIF4F. The eIF4F complex is an important part of the protein synthesis machinery that produces growth factors, cyclins and other signaling proteins and receptors crucial for cell growth, angiogenesis and proliferation. mTORC2 activates serum- and glucocorticoid-regulated kinase (SGK) and protein kinase C (PKC) by phosphorylation, leading to cell cycle regulation and survival as well as reorganization of the cell cytoskeleton.

strategy could be adopted for tackling the cellular abnormalities seen in airway remodeling and for tumor growth.

Zileuton is an upstream inhibitor of the leukotrienestimulated PI3K/Akt/mTOR signaling. Knowing the downstream molecular players involved in the signal pathways will allow us to be more specific in selecting targets for drug intervention. The large percentage of non- 
responders in antileukotriene therapy [8] may be due to genetic or epigenetic variations among asthma sufferers; these variations may result in differences in the quantity or the properties of the downstream enzymes. Targeting specific enzymes may therefore reduce the pool of nonresponders in asthma therapy. Caution must be exercised, however, in interpreting the data from Chen et al. [2]. They did not demonstrate that the observed airway remodeling is directly mediated by leukotrienes. The leu- kotriene antagonist could reduce inflammation and it could be that the remodeling is a downstream event driven by one or many of the inflammatory mediators released secondary to leukotriene release. This possibility needs to be addressed in future experiments. Furthermore, the pathways identified in the rat model need to be explored in human tissues, to verify that indeed the same pathways are altered when humans are exposed to inhibitors of leukotriene formation.

\section{References}

$>1$ Louis R, Schleich F, Barnes PJ: Corticosteroids: still at the frontline in asthma treatment? Clin Chest Med 2012;33:531-541.

$\checkmark 2$ Chen WJ, Liaw SF, Lin CC, Lin MW, Chang FT: Effects of zileuton on airway smooth muscle remodeling after repeated allergen challenge in brown Norway rats. Respiration 2013; 86:421-429.

$\checkmark 3$ Gomez-Pinillos A, Ferrari AC: mTOR signaling pathway and mTOR inhibitors in cancer therapy. Hematol Oncol Clin North Am 2012; 26:483-505.

4 Khan KH, Yap TA, Yan L, Cunningham D: Targeting the PI3K-AKT-mTOR signaling network in cancer. Chin J Cancer 2013;32: 253-265.

5 Dahlén SE: Treatment of asthma with antileukotrienes: first line or last resort therapy? Eur J Pharmacol 2006;533:40-56.

6 Berger W, De Chandt MT, Cairns CB : Zileuton: clinical implications of 5-Lipoxygenase inhibition in severe airway disease. Int J Clin Pract 2007;61:663-676.

7 Scow DT, Luttermoser GK, Dickerson KS: Leukotriene inhibitors in the treatment of allergy and asthma. Am Fam Physician 2007;75: 65-70.
-8 Terashima T, Amakawa K, Matsumaru A, Yamaguchi K: Correlation between cysteinyl leukotriene release from leukocytes and clinical response to a leukotriene inhibitor. Chest 2002;122:1566-1570.

-9 Burgess JK, Lee JH, Ge Q, Ramsay EE, Poniris MH, Parmentier J, Roth M, Johnson PR, Hunt NH, Black JL, Ammit AJ: Dual ERK and phosphatidylinositol 3-kinase pathways control airway smooth muscle proliferation: differences in asthma. J Cell Physiol 2008;216:673679.

10 Lazaris-Karatzas A, Montine KS, Sonenberg $\mathrm{N}$ : Malignant transformation by a eukaryotic initiation factor subunit that binds to mRNA 5 ' cap. Nature 1990;345:544-547.

-11 Schmelzle T, Hall MN: TOR, a central controller of cell growth. Cell 2000;103:253262.

12 Zhou L, Goldsmith AM, Bentley JK, Jia Y, Rodriguez ML, Abe MK, Fingar DC, Hershenson $\mathrm{MB}$ : 4E-binding protein phosphorylation and eukaryotic initiation factor-4E release are required for airway smooth muscle hypertrophy. Am J Respir Cell Mol Biol 2005;33:195202.
13 Roth M, Johnson PR, Borger P, Bihl MP, Rüdiger JJ, King GG, Ge Q, Hostettler K, Burgess JK, Black JL, Tamm M: Dysfunctional interaction of C/EBPalpha and the glucocorticoid receptor in asthmatic bronchial smooth-muscle cells. N Engl J Med 2004;351: 560-574.

14 Gombart AF, Hofmann WK, Kawano S, Takeuchi S, Krug U, Kwok SH, Larsen RJ, Asou H, Miller CW, Hoelzer D, Koeffler HP: Mutations in the gene encoding the transcription factor CCAAT/enhancer binding protein alpha in myelodysplastic syndromes and acute myeloid leukemias. Blood 2002;99: 1332-1340.

15 Sekine O, Nishio Y, Egawa K, Nakamura T, Maegawa H, Kashiwagi A: Insulin activates CCAAT/enhancer binding proteins and proinflammatory gene expression through the phosphatidylinositol 3-kinase pathway in vascular smooth muscle cells. J Biol Chem 2002;277:36631-36639.

16 Wang GL, Iakova P, Wilde M, Awad S, Timchenko NA: Liver tumors escape negative control of proliferation via PI3K/Akt-mediated block of C/EBP alpha growth inhibitory activity. Genes Dev 2004;18:912-925. 